\title{
MicroRNA-142-3p relieves neuropathic pain by targeting high mobility group box 1
}

\author{
YANG ZHANG $^{1 *}, \mathrm{JUNYING} \mathrm{MOU}^{2 *}, \mathrm{LI} \mathrm{CAO}^{3 *}, \mathrm{SU}_{\mathrm{ZHEN}}{ }^{1}, \mathrm{HONGJUAN} \mathrm{HUANG}^{4}$ and HONGGUANG BAO ${ }^{5}$ \\ ${ }^{1}$ Department of Anesthesiology, Huai'an First People's Hospital, Nanjing Medical University, Huai'an, \\ Jiangsu 223300; ${ }^{2}$ Department of Anesthesiology, The Central Hospital of Enshi Autonomous Prefecture, \\ Enshi Clinical College of Wuhan University, Enshi, Hubei 445000; ${ }^{3}$ Department of Internal Medicine, \\ Suizhou Zengdu Hospital, Suizhou, Hubei 441300; ${ }^{4}$ Department of Neurology, Huai'an Second People's Hospital and \\ The Affiliated Huai'an Hospital of Xuzhou Medical University, Huai'an, Jiangsu 223002; ${ }^{5}$ Department of Anesthesiology, \\ Nanjing First Hospital, Nanjing Medical University, Nanjing, Jiangsu 210006, P.R. China
}

Received February 13, 2017; Accepted October 6, 2017

DOI: $10.3892 /$ ijmm.2017.3222

\begin{abstract}
MicroRNA (miRNA) are emerging as critical regulators of neuropathic pain development. Neuroinflammation contributes to the development of neuropathic pain. miR-142-3p has been characterized as an inflammation-related miRNA in various pathological processes. However, little is known about the role of miR-142-3p in neuroinflammation and neuropathic pain. The present study aimed to investigate the function of miR-142-3p in neuropathic pain by creating a murine model using spinal nerve ligation (SNL). A significant reduction in miR-142-3p expression was observed in the dorsal root ganglion of mice with SNL $(\mathrm{P}<0.05)$ compared with control mice. Overexpression of miR-142-3p significantly inhibited neuropathic pain and neuroinflammation in mice with SNL $(\mathrm{P}<0.05)$. High mobility group box 1 (HMGB1) was identified as a direct target gene of miR-142-3p by bioinformatic analysis and dual-luciferase reporter assays. Overexpression of miR-142-3p significantly reduced the mRNA and protein expression levels of HMGB1 in vitro and in vivo $(\mathrm{P}<0.05)$. In
\end{abstract}

Correspondence to: Dr Hongguang Bao, Department of Anesthesiology, Nanjing First Hospital, Nanjing Medical University, 68 Changle Road, Nanjing, Jiangsu 210006, P.R. China

E-mail: hongguang_bao@hotmail.com

Dr Hongjuan Huang, Department of Neurology, Huai'an Second People's Hospital and The Affiliated Huai'an Hospital of Xuzhou Medical University, 62 South Huaihai Road, Huai'an, Jiangsu 223002, P.R. China E-mail: hongjuanhuanghj@163.com

*Contributed equally

Abbreviations: HMGB1, high mobility group box 1; miRNA, microRNA; NF- $\kappa \mathrm{B}$, nuclear factor- $\kappa \mathrm{B}$; SNL, spinal nerve ligation; DRG, dorsal root ganglion; IL, interleukin; TNF, tumor necrosis factor; UTR, untranslated region

Key words: high mobility group box 1, neuroinflammation, miR-142-3p, neuropathic pain addition, HMGB1 mRNA expression and miR-142-3p expression were inversely correlated in mice with SNL. Furthermore, overexpression of HMGB1 significantly reversed the inhibitory effect of miR-142-3p on neuroinflammation and neuropathic pain development $(\mathrm{P}<0.05)$. Overall, these results suggest that miR-142-3p functions as a negative regulator of neuropathic pain development through the downregulation of HMGB1, indicating that miR-142-3p may serve as a potential therapeutic target for neuropathic pain.

\section{Introduction}

Neuropathic pain is a chronic disorder characterized by hyperalgesia, allodynia and spontaneous pain affecting the somatosensory system (1). Neuropathic pain has emerged as a complicated and refractory disease affecting an increasing number of people worldwide $(2,3)$. Unfortunately, there remains a lack of effective drugs to target and prevent neuropathic pain. Although conventional drugs, including opioids and non-steroidal anti-inflammatory drugs, have been used to prevent neuropathic pain, the therapeutic effect is not fully satisfactory (4). Therefore, it is essential to investigate the molecular mechanism underlying physiological and pathological pain status, and to develop novel and effective therapeutic strategies.

MicroRNA (miRNA), a group of endogenous, small and non-coding RNA, have emerged as critical regulators of gene expression (5). miRNA post-transcriptionally regulate gene expression by binding to the 3'-untranslated region (3'-UTR) of target mRNA, inducing mRNA instability and degradation (5). Research has demonstrated that miRNA expression is dysregulated in various pathological diseases, such as neuropathic pain (6-9). The targeting of miRNA has indicated promising results in preventing neuropathic pain development in animal models $(10,11)$. Therefore, an improved understanding of the role of miRNA in neuropathic pain may help in developing novel therapeutic approaches for various diseases.

A previous study has demonstrated that the spinal cord is pivotal in pain perception and modulation (12). Glial activation and neuronal sensitization in the spinal cord contributes to pain 
hypersensitivity (13). Astrocytes and microglia are activated in the spinal cord dorsal horn following nerve injury, leading to persistent neuroinflammation and pain hypersensitivity (14). The release of potent inflammatory mediators, including tumor necrosis factor- $\alpha$ (TNF- $\alpha$ ), interleukin-1 $\beta$ (IL-1 $\beta$ ) and IL-6, contributes to pain hypersensitivity and persistent pain development $(15,16)$.

High mobility group box 1 (HMGB1) is an ubiquitous non-histone DNA-binding protein that is a critical regulator of inflammation (17). Various studies have indicated that HMGB1 is involved in inflammation-related diseases, including sepsis (18) and osteoarthritis $(19,20)$. HMGB1 may engage with advanced glycation end products, Toll-like receptors or integrin to provoke an inflammatory reaction (21-23). HMGB1 is abundantly expressed in various cell types, including neurons and glia (24). The injection of HMGB1 into the sciatic nerve of rats induces neuropathic pain-like behavior (25). HMGB1 is induced in the dorsal root ganglion (DRG) following peripheral nerve injury (26). Inhibition of HMGB1 effectively alleviates neuroinflammation and neuropathic pain development in animal models (27). Therefore, HMGB-1 has been suggested as a therapeutic target for the treatment of neuropathic pain $(28,29)$.

A previous study has suggested that miR-142-3p is a novel regulator of inflammation that negatively regulates proinflammatory mediators, including nuclear factor- $\kappa \mathrm{B}(\mathrm{NF}-\kappa \mathrm{B}), \mathrm{TNF}-\alpha$ and IL-6 in macrophages (30). Overexpression of miR-142-3p in human myeloid inflammatory cells inhibits the production of inflammatory mediators, including TNF- $\alpha$ and IL-6 (31). A recent study demonstrated that miR-142-3p inhibits inflammation in chondrocytes of osteoarthritic conditions (32). These studies suggest an anti-inflammatory role for miR-142-3p. However, whether miR-142-3p participates in the neuroinflammation of neuropathic pain remains unknown.

The present study demonstrated that miR-142-3p was reduced in the DRG of mice following spinal nerve ligation (SNL) injury. Overexpression of miR-142-3p inhibited neuropathic pain and neuroinflammation. HMGB1 was identified as a target gene of miR-142-3p. Overexpression of miR-142-3p inhibited HMGB1 expression in vitro and in vivo, while an inverse correlation was observed between HMGB1 mRNA and miR-142-3p expression in vivo. Additionally, overexpression of HMGB1 significantly reversed the protective effects of miR-142-3p. Taken together, these results suggest that miR-142-3p inhibits neuropathic pain development through downregulation of HMGB1-mediated proinflammation, which may serve as a potential therapeutic target for the treatment of neuropathic pain.

\section{Materials and methods}

Animals. A total of 90 adult male 8 -week-old ICR mice weighing 20-25 g were purchased from the Laboratory Animal Center of Nanjing Medical University (Nanjing, China). The mice were housed in a 12-h light/dark cycle at a room temperature of $22 \pm 1^{\circ} \mathrm{C}$ and humidity of $50-60 \%$ with ad libitum access to water and food. All animal experimental procedures were performed in accordance with the guidelines of the International Association for the Study of Pain and the National Institute of Health Guide for the Care and Use of Laboratory Animals (NIH Publications no. 80-23, revised 1996). The present study was reviewed and approved by the local Institutional Animal Care and Use Committee of Nanjing Medical University.

Cell culture. The DRG was cultured as previously described (33). Briefly, the bilateral L3 and L4 DRG were rapidly dissected from mice $(\mathrm{n}=3)$ and digested with $1 \mathrm{mg} / \mathrm{ml}$ trypsin and $2 \mathrm{mg} / \mathrm{ml}$ collagenase (Sigma-Aldrich; Merck KGaA, Darmstadt, Germany) in Dulbecco's modified Eagle's medium (DMEM; Gibco; Thermo Fisher Scientific, Inc., Waltham, MA, USA). After mechanical dissociation into single cells using a Pasteur pipette, the DRG neurons were seeded into 6 -well plates at a density of $5 \times 10^{5}$ cells/well in DMEM for $24 \mathrm{~h}$ at $37^{\circ} \mathrm{C}$ with $5 \% \mathrm{CO}_{2}$. The seeded cells were treated with $5 \mu \mathrm{g} / \mathrm{ml}$ cytarabine (Sigma-Aldrich; Merck KGaA) to repress the growth of non-neuronal cells. Following this, the cells were grown in DMEM/F-12 supplemented with $10 \%$ fetal bovine serum (FBS) (both from Gibco; Thermo Fisher Scientific, Inc.), $10 \mathrm{ng} / \mathrm{ml}$ nerve growth factor (Invitrogen; Thermo Fisher Scientific, Inc.), $0.1 \mathrm{mg} / \mathrm{ml} \mathrm{L-glutamine} \mathrm{and} 1 \%$ penicillin/streptomycin mix (Sigma-Aldrich; Merck KGaA). The medium was refreshed twice a week. 293T cells were obtained from the Cell Bank of the Chinese Academy of Sciences (Shanghai, China) and cultured in DMEM supplemented with 10\% FBS and $1 \%$ penicillin/streptomycin mix. All cells were cultured in a humidified atmosphere of $5 \% \mathrm{CO}_{2}$ at $37^{\circ} \mathrm{C}$.

SNL model of neuropathic pain. Mice $(n=45)$ were anesthetized by peritoneal injection of $50 \mathrm{mg} / \mathrm{kg}$ sodium pentobarbital (Merck KGaA). Following incision of the skin, the paraspinal muscles were bluntly dissected. The L5 transverse process was removed to expose the L3 and L4 spinal nerves. The exposed L4 spinal nerve was tightly ligated with a 6-0 silk suture and transected distal to the ligation. In sham mice $(n=15)$, the L5 transverse process was exposed, and the L4 spinal nerve was not touched and ligated. SNL mice were divided into two groups: SNL + lentiviral vector (LV)-negative control (NC) $(n=15)$ and SNL + LV-miR-142-3p $(n=15)$.

Intrathecal catheter implantation and injection. Following anesthesia, the occipital muscles of mice were separated to expose the cisternal membrane. A polyethylene catheter (PE-10; Braintree Scientific, Inc., Braintree, MA, USA) was inserted through an incision in the cisterna magna. The proper location of the intrathecal implantation was validated by bilateral hind limb paralysis with injection of $2 \%$ lidocaine $(1 \mu \mathrm{l})$ (SigmaAldrich; Merck KGaA). Animals that failed to display paralysis by lidocaine were not included in the experiments. Intrathecal delivery of recombinant LV-miR-142-3p [8x10 units (TU); $1 \mu \mathrm{l})]$ or LV-NC (8x10 5 TU; $1 \mu \mathrm{l})$ or HMGB1 small interfering RNA (siRNA) $(50 \mu \mathrm{g}, 1 \mu \mathrm{l})$ or NC siRNA $(50 \mu \mathrm{g}$, $1 \mu \mathrm{l}$ ) (Shanghai GenePharma Co., Ltd., Shanghai, China) was performed by a microinjection syringe $(10 \mu \mathrm{l})$ linked with the intrathecal catheter. The sequence of the HMGB1 siRNA was 5'-CUCGUUAUGAAAGAGAAAUTT-3' and of the NC siRNA was 5'-UUCUCCGAACGUGUCACGUTT-3'.

Assessment of thermal hyperalgesia. Thermal hyperalgesia was assessed by measuring the paw withdrawal latencies in response to radiant heat stimulation using a Plantar Analgesia Meter (IITC Life Science, Inc., Woodland Hills, CA, USA). The 
mouse was placed in a plastic chamber with a glass floor upon a radiant heat source. After acclimatization to the environment for $1 \mathrm{~h}$, the radiant heat source was launched and the duration between the start and paw withdrawal was recorded by a digital timer. A cut-off time was set at $20 \mathrm{sec}$ to avoid tissue damage and the measurement was repeated five times for each mouse. A thermal stimulus was delivered at 5-min intervals.

Assessment ofmechanical allodynia.Mechanical allodynia was assessed by measuring the paw withdrawal threshold (PWT) in response to Von Frey hair (IITC Life Science, Inc.) stimulation. The mouse was placed in a plastic cage with a plexiglass floor. After acclimatization to the environment for $1 \mathrm{~h}$, the mouse was subjected to an ascending series of Von Frey hairs. Brisk withdrawal or paw flinching upon stimulus was defined as a positive response. The Von Frey hairs were maintained for 5-6 sec at 5-min intervals and the measurement was repeated five times for each mouse.

Reverse transcription-quantitative polymerase chain reaction (RT-qPCR). Total RNA from DRG was extracted using TRIzol reagent (Invitrogen; Thermo Fisher Scientific, Inc.), according to standard protocols. For detection of miRNA expression, qPCR was performed using a TaqMan miRNA assay kit (Ambion; Thermo Fisher Scientific, Inc.) and U6 served as the endogenous control. For detection of mRNA expression, cDNA was synthesized by M-MLV reverse transcriptase (BioTeke Corp., Beijing, China) and qPCR amplification was performed using a SYBR-Green system (Applied Biosystems; Thermo Fisher Scientific, Inc.). The primer sequences used in the present study were as follows: miR-142-3p, forward, 5'-TGCGGTGTAGTGT TTCCTACTT-3' and reverse, 5'-CCAGTGCAGGGTCCGA GGT-3'; HMGB1 forward, 5'-GCTGACAAGGCTCGTTAT GAA-3' and reverse, 5'-CCTTTGATTTTGGGGCGGTA-3'; U6 forward, 5'-TTGGTCTGATCTGGCACATATAC-3' and reverse, 5'-AAAAATATGGAGCGCTTCACG-3'; glyceraldehyde 3-phosphate dehydrogenase (GAPDH) forward, 5'-AGGTCG GTGTGAACGGATTTG-3' and reverse, 5'-GGGGTCGTTGA TGGCA ACA-3'. The thermal cycling conditions were set as $95^{\circ} \mathrm{C}$ for $3 \mathrm{~min}$, followed by 40 cycles of $95^{\circ} \mathrm{C}$ for $15 \mathrm{sec}$ and $60^{\circ} \mathrm{C}$ for $30 \mathrm{sec}$. GAPDH served as the endogenous control. Relative gene expression was determined using the $2^{-\Delta \Delta \mathrm{Cq}}$ method (34).

Enzyme-linked immunosorbent assay (ELISA). Levels of TNF- $\alpha$, IL-1 $\beta$, IL-6 and IL-10 in the L3-L5 DRG were determined using commercial ELISA kits, including TNF- $\alpha$ Quantikine ELISA kit (cat. no. MTA00B), IL-1 $\beta$ Quantikine ELISA kit (cat.no. MLB00C), IL-6 Quantikine ELISA kit (cat.no. M6000B) and IL-10 Quantikine ELISA kit (cat. no. M1000B), purchased from R\&D Systems, Inc. (Minneapolis, MN, USA) according to the manufacturer's instructions.

Dual-luciferase reporter assay. miRNA targets were predicted using the algorithms of TargetScan (targetscan.org). A fragment of the HMGB1 3'-UTR containing either the predicted seed-matched or mutant sequences of miR-142-3p was inserted into pmirGLO Dual-Luciferase miRNA Target Expression Vector (Promega Corp., Madison, WI, USA). 293T cells were co-transfected with pmirGLO vector with miR-142-5p mimics (Shanghai GenePharma Co., Ltd.) using Lipofectamine 2000
(Invitrogen; Thermo Fisher Scientific, Inc.) and incubated for $48 \mathrm{~h}$. Relative luciferase activity was detected by a dual-luciferase reporter system (Promega Corp.). Relative luciferase activity was calculated by the formula: Firefly luciferase activity/Renilla luciferase activity.

Western blot analysis. Proteins were exacted from DRG using radioimmunoprecipitation assay buffer (Sigma-Aldrich; Merck KGaA). Protein concentrations were measured using a bicinchoninic acid kit (Beyotime Institute of Biotechnology, Haimen, China). Samples of $50 \mu \mathrm{g}$ total protein were separated using $10 \%$ sodium dodecyl sulfate-polyacrylamide gel electrophoresis (SDS-PAGE) and transferred onto polyvinylidene fluoride membranes (EMD Millipore, Billerica, MA, USA). The membranes were then blocked by $5 \%$ non-fat dry milk for $1 \mathrm{~h}$ at $37^{\circ} \mathrm{C}$, and subsequently incubated overnight at $4^{\circ} \mathrm{C}$ with primary antibodies, including rabbit monoclonal anti-HMGB1 (cat. no. ab79823; 1:10,000), rabbit monoclonal anti-phosphorylated (p)-p65 (cat. no. ab86299; 1:2,000) and rabbit polyclonal anti-GAPDH (cat. no. ab9485; 1:2,500) (Abcam, Cambridge, UK). Following incubation with horseradish peroxidase-conjugated secondary antibody (goat anti-rabbit immunoglobulin G; cat. no. ab6721; 1:2,000; Abcam) for $2 \mathrm{~h}$ at room temperature, the protein bands were detected by enhanced chemiluminescence reagents (Pierce; Thermo Fisher Scientific, Inc.). The protein band intensities were quantified with densitometry using Image-Pro Plus 6.0 software (Media Cybernetics, Inc., Rockville, MD, USA).

Statistical analysis. Data were expressed as the mean \pm standard deviation. Statistical analyses were performed using the Student's t-test or one-way analysis of variance followed by the Bonferroni post hoc test using SPSS 18.0 software (SPSS, Inc., Chicago, IL, USA). The correlation between miR-142-3p expression and HMGB1 mRNA expression was determined using Spearman's rank correlation test. $\mathrm{P}<0.05$ was considered to indicate a statistically significant difference.

\section{Results}

miR-142-3p is downregulated in the DRG of mice with SNL. To investigate the potential role of miR-142-3p in neuropathic pain, its expression status was characterized in the DRG of mice with SNL using RT-qPCR. The results demonstrated that miR-142-3p was significantly downregulated in mice with SNL compared with the levels in the sham group by postoperative days 3, 7, 14 and 21 (Fig. 1A). The results indicate that downregulation of miR-142-3p may be an important event in the development and maintenance of neuropathic pain.

Overexpression of miR-142-3p relieves neuropathic pain in mice with $S N L$. To investigate the precise function of miR-142-3p in regulating neuropathic pain, gain-of-function experiments were performed by intrathecal injection of LV-miR-142-3p. Infection with LV-miR-142-3p significantly upregulated miR-142-3p expression level in the DRG of mice with SNL compared with the level in the SNL + LV-NC group (Fig. 1B). The effect of miR-142-3p overexpression on neuropathic pain development was investigated by assessment of thermal hyperalgesia and mechanical allodynia. The results 

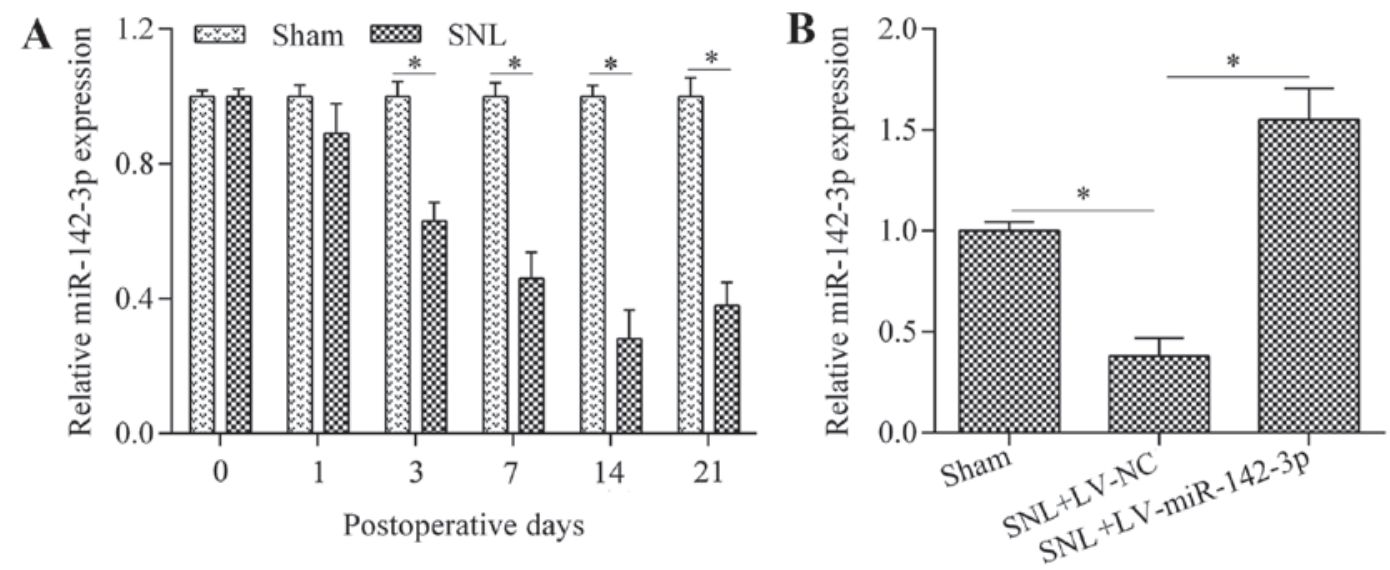

Figure 1. Expression of miR-142-3p in the DRG of mice with SNL. (A) The expression of miR-142-3p in the L3-L5 DRG of mice was detected by RT-qPCR $(\mathrm{n}=3)$. (B) RT-qPCR analysis of miR-142-3p expression in the L3-L5 DRG of mice infected with LV-miR-142-3p or the LV-NC on postoperative day 14 (n=3). " $\mathrm{P}<0.05$ as indicated. miR, microRNA; DRG, dorsal root ganglion; SNL, spinal nerve ligation; RT-qPCR, reverse transcription-quantitative polymerase chain reaction; $\mathrm{NC}$, negative control; $\mathrm{LV}$, lentiviral vector.
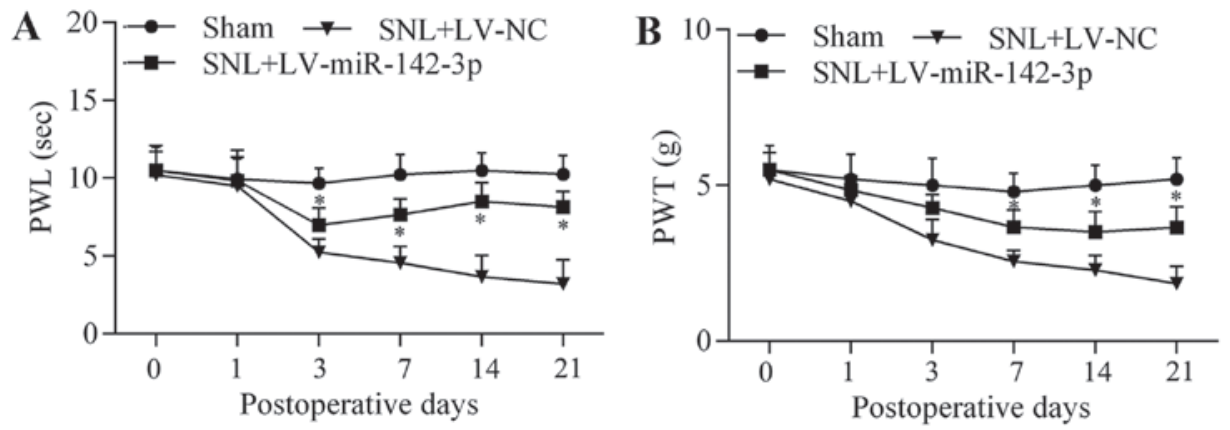

Figure 2. Overexpression of miR-142-3p relieves neuropathic pain in mice with SNL. (A) Thermal hyperalgesia was assessed by measuring the PWL in response to radiant heat stimulation. (B) Mechanical allodynia was assessed by measuring the PWT in response to Von Frey hair stimulation. $\mathrm{n}=6$. ${ }^{*} \mathrm{P}<0.05 \mathrm{vs}$. SNL $+\mathrm{LV}-\mathrm{NC}$. miR, microRNA; SNL, spinal nerve ligation; PWL, paw withdrawal latency; PWT, paw withdrawal threshold; NC, negative control; LV, lentiviral vector.

demonstrated that overexpression of miR-142-3p significantly increased paw withdrawallatency (PWL) (Fig. 2A) and PWT (Fig. 2B) in LV-miR-142-3p-infected SNL mice compared with that in LV-NC-infected SNL mice at postoperative days $3,7,14$ and 21 , indicating that overexpression of miR-142-3p relieved thermal hyperalgesia and mechanical allodynia. These results suggest that miR-142-3p functions as a negative regulator of neuropathic pain.

Overexpression of miR-142-3p suppresses neuroinflammation in mice with SNL. To further explore the biological function of $\mathrm{miR}-142-3 \mathrm{p}$ in regulating neuropathic pain, the effect of miR-142-3p overexpression on neuroinflammation was investigated by measuring proinflammatory cytokines (TNF- $\alpha$, IL-1 $\beta$ and IL-6) and the IL-10 anti-inflammatory cytokine. The results indicated that miR-142-3p overexpression in mice with SNL was associated with a significant reduction in the expression levels of TNF- $\alpha$ (Fig. 3A), IL-1 $\beta$ (Fig. 3B) and IL-6 (Fig. 3C), and an increase in the expression level of IL-10 (Fig. 3D) compared with the levels in the SNL + LV-NC group. These data suggest an inhibitory role of miR-142-3p on neuroinflammation in mice with SNL.

$H M G B 1$ is a direct target gene of miR-142-3p. To investigate the molecular mechanism underlying miR-142-3p-induced inhibi- tory effect on neuropathic pain and neuroinflammation, predicted targets of miR-142-3p were sought by established miRNA target prediction programs. Notably, it was revealed that HMGB1, a critical gene of neuropathic pain and inflammation (26), was a predicted target gene of miR-142-3p (Fig. 4A). This prediction was confirmed by dual-luciferase reporter assays. The putative seed-matched or mutant sequences of miR-142-3p for the HMGB1 3'-UTR were cloned into the luciferase reporter vector. Overexpression of miR-142-3p significantly decreased the luciferase activity of the reporter vector containing the 3'-UTR of HMGB1 compared with the activity in the NC group (Fig. 4B). However, miR-142-3p overexpression demonstrated no significant effect on the reporter vector containing the mutant HMGB1 3'-UTR compared with the NC group (Fig. 4B). Furthermore, the effect of miR-142-3p overexpression on HMGB1 expression was investigated in cultured DRG neurons. The results indicated that miR-142-3p overexpression significantly decreased the mRNA (Fig. 4C) and protein (Fig. 4D) expression levels of HMGB1 in DRG neurons compared with the levels in the NC group. Overall, these results suggest that HMGB1 is a direct target gene of miR-142-3p.

$H M G B 1$ is negatively correlated with miR-142-3p in mice with $S N L$. To further confirm the targeted relationship between miR-142-3p and HMGB1, the correlation between miR-142-3p 

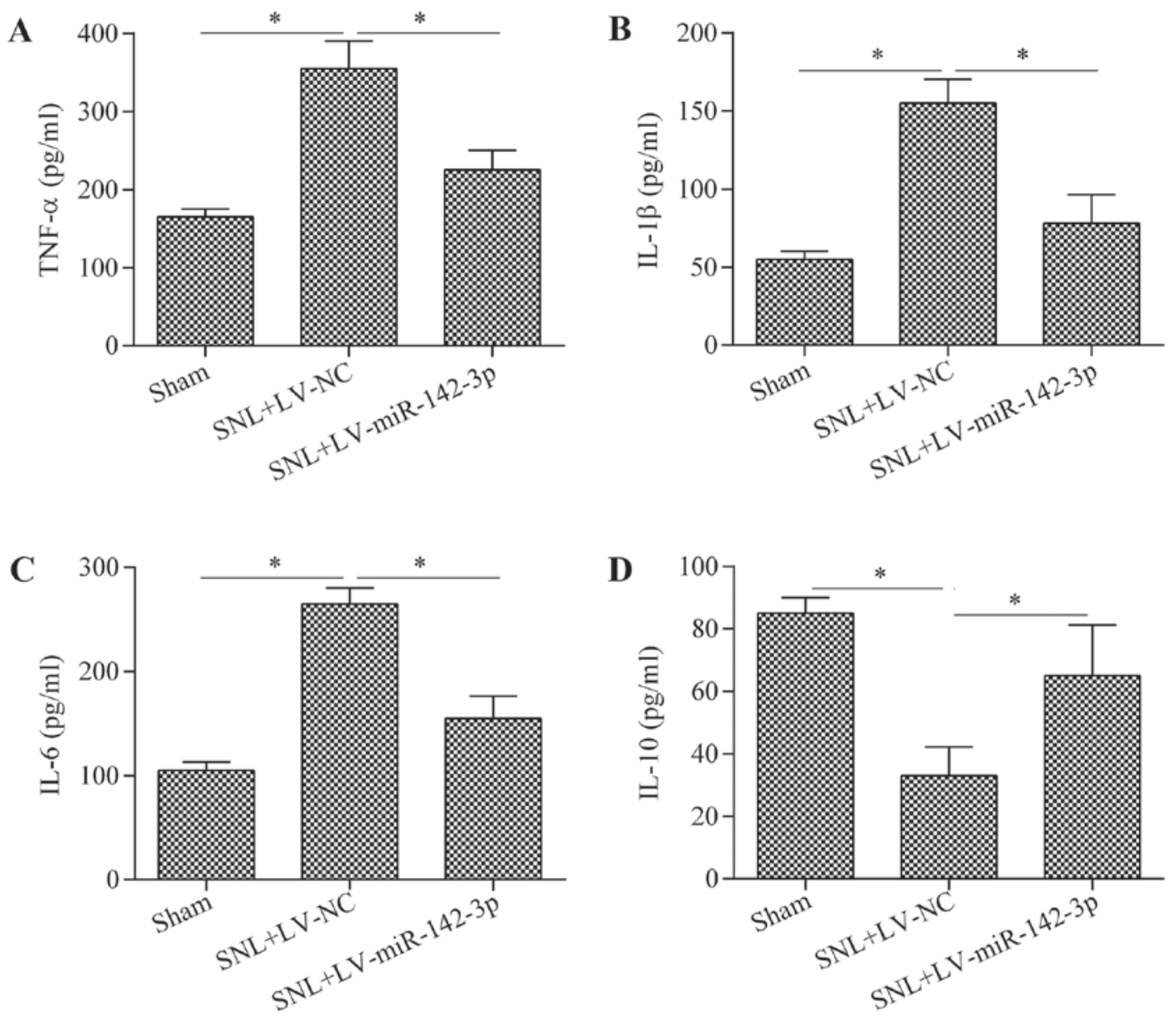

Figure 3. Overexpression of miR-142-3p suppresses neuroinflammation in mice with SNL. Protein expression levels of (A) TNF- $\alpha$, (B) IL-1 $\beta$, (C) IL-6 and (D) IL-10 in the L3-L5 DRG of mice were determined by ELISA on postoperative day 14. $\mathrm{n}=3$. ${ }^{*} \mathrm{P}<0.05$ as indicated. miR, microRNA; SNL, spinal nerve ligation; TNF, tumor necrosis factor; IL, interleukin; DRG, dorsal root ganglion; NC, negative control; LV, lentiviral vector; ELISA, enzyme-linked immunosorbent assay.

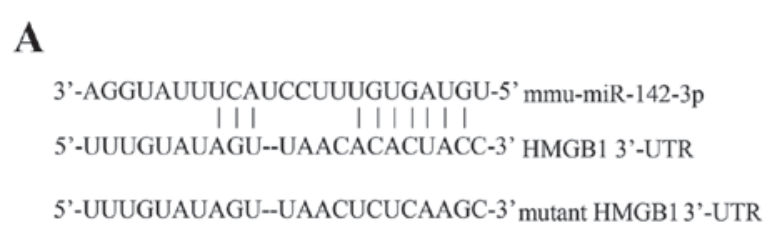

B

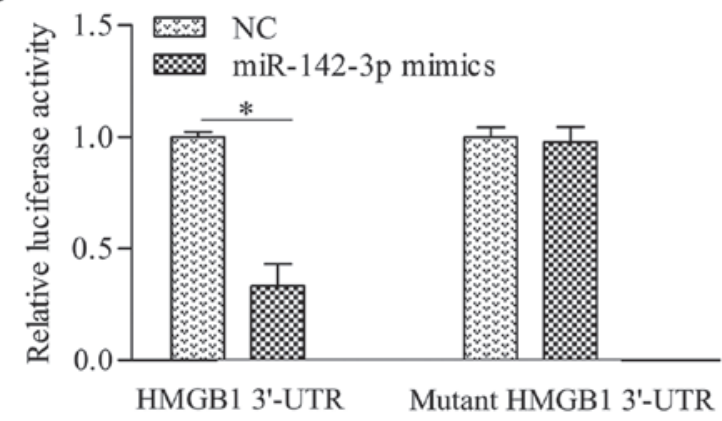

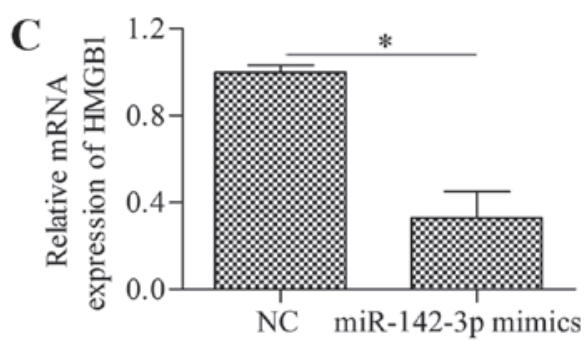

D

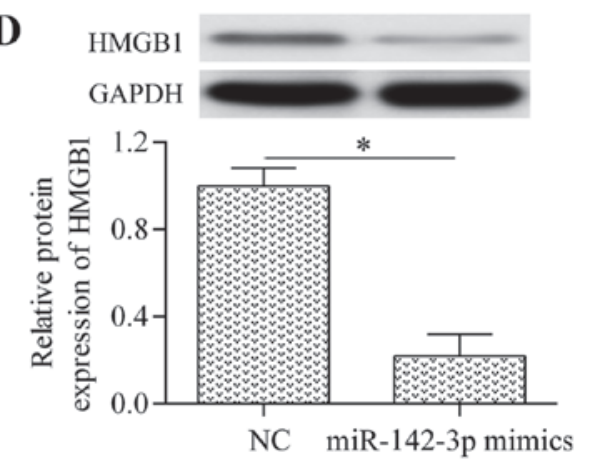

Figure 4. HMGB1 is a target gene of miR-142-3p. (A) Predicted miR-142-3p target sequences in the 3'-UTR of HMGB1 mRNA. (B) Dual-luciferase reporter assay of $293 \mathrm{~T}$ cells co-transfected with the reporter vector and miR-142-3p mimics for $48 \mathrm{~h}$. (C) Reverse transcription-quantitative polymerase chain reaction analysis of HMGB1 mRNA expression in cultured DRG neurons transfected with miR-142-3p mimics for $48 \mathrm{~h}$. (D) Western blot analysis of HMGB1 protein expression in cultured DRG neurons transfected with miR-142-3p mimics for $48 \mathrm{~h} . \mathrm{n}=3$. " $\mathrm{P}<0.05$ as indicated. miR, microRNA; HMGB1, high mobility group box 1; UTR, untranslated region; DRG, dorsal root ganglion; NC, negative control; LV, lentiviral vector. 

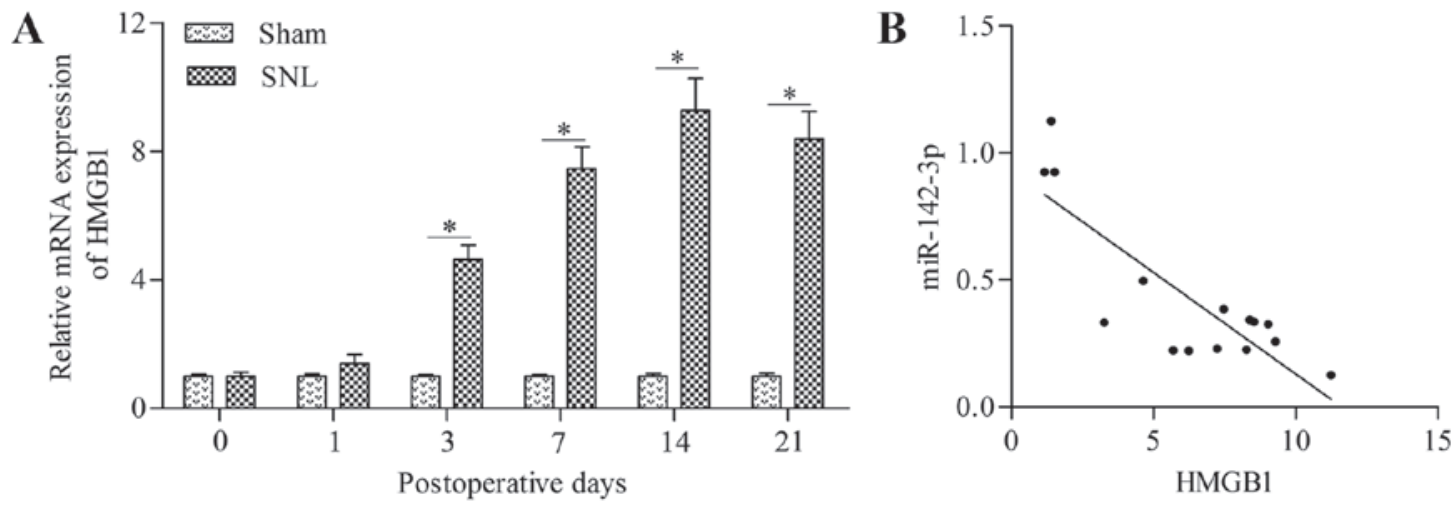

Figure 5. HMGB1 is negatively correlated with miR-142-3p in mice with SNL. (A) Reverse transcription-quantitative polymerase chain reaction analysis of HMGB1 mRNA expression in L3-L5 DRG of mice $(n=3)$. P $<0.05$ as indicated. (B) Correlation between HMGB1 mRNA expression and miR-142-3p expression in the DRG of mice with SNL was analyzed by a Spearman's rank correlation test. $\mathrm{n}=15 ; \mathrm{r}=-0.5898 ; \mathrm{P}<0.05$. miR, microRNA; HMGB1, high mobility group box 1; SNL, spinal nerve ligation; DRG, dorsal root ganglion.
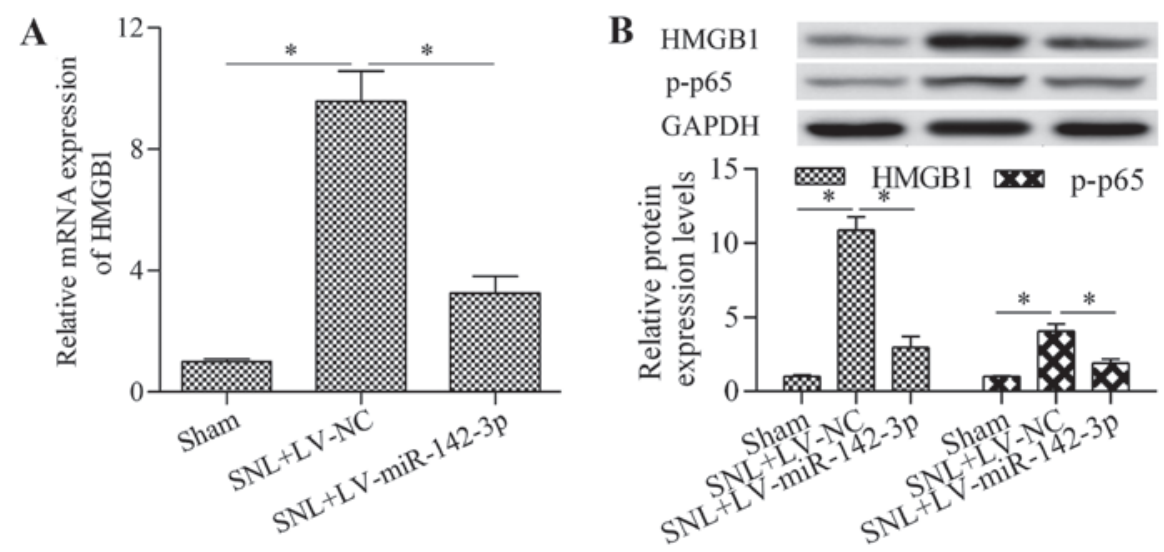

Figure 6. Overexpression of miR-142-3p inhibits HMGB1 expression in mice with SNL. (A) Reverse transcription-quantitative polymerase chain reaction analysis of HMGB1 mRNA expression in the DRG of mice with SNL infected with LV-miR-142-3p on postoperative day 14. (B) Western blot analysis of HMGB1 and p-p65 protein expression in the DRG of mice with SNL infected with LV-miR-142-3p on postoperative day 14. $\mathrm{n}=3$. "P $<0.05$ as indicated. miR, microRNA; HMGB1, high mobility group box 1; SNL, spinal nerve ligation; DRG, dorsal root ganglion; NC, negative control; LV, lentiviral vector; $p$, phosphorylated.

and HMGB1 in mice with SNL was analyzed. The results demonstrated that the HMGB1 mRNA expression level was significantly upregulated in the DRG of mice with SNL at postoperative days $3,7,14$ and 21 compared with the levels in the sham group (Fig. 5A). Furthermore, HMGB1 demonstrated an inverse correlation with miR-142-3p expression (Fig. 5B). These data suggest an inverse relationship between miR-142-3p and HMGB1 mRNA expression in mice with SNL.

Overexpression of miR-142-3p inhibits HMGB1 expression in mice with $S N L$. To investigate whether miR-142-3p regulates HMGB1 expression in vivo, the present study detected the effect of miR-142-3p overexpression on HMGB1 expression in mice with SNL. The results demonstrated that overexpression of miR-142-3p significantly inhibited HMGB1 mRNA (Fig. 6A) and protein (Fig. 6B) expression levels in the DRG of mice with SNL compared with the levels in the SNL + LV-NC group. Furthermore, the levels of p-NF- $\mathrm{BB}$ p65 protein in mice with SNL were also significantly reduced by miR-142-3p overexpression compared with the levels in the SNL + LV-NC group (Fig. 6B). To confirm whether HMGB1 was involved in regulating neuroinflammation in mice with SNL, HMGB1 expression was inhibited by transfection of HMGB1 siRNA.
The results demonstrated that treatment with HMGB1 siRNA significantly reduced the expression of HMGB1 compared with the levels in the SNL + NC siRNA group (Fig. 7A). Additionally, the expression levels of IL-1 $\beta$ (Fig. 7B), TNF- $\alpha$ (Fig. 7C) and IL-6 (Fig. 7D) were significantly downregulated by HMGB1 siRNA compared with the levels in the SNL + NC siRNA group. Overall, these data suggest that miR-142-3p regulates neuroinflammation in mice with SNL through the HMGB1/NF-kB/inflammatory cytokine production pathway.

Overexpression of HMGB1 reverses the inhibitory effect of miR-142-3p on neuropathic pain and neuroinflammation. To further investigate whether miR-142-3p alleviates neuropathic pain through targeting HMGB1, rescue experiments were performed. Mice were infected with LV-miR-142-3p and LV-HMGB1. Results demonstrated that infection with LV-HMGB1 significantly restored the decreased HMGB1 expression in LV-miR-142-3p-infected mice with SNL compared with the level in the SNL + LV-miR-142-3p group (Fig. 8A). As expected, the regulatory effects of miR-142-3p overexpression on IL-1 $\beta$ (Fig. 8B), IL-6 (Fig. 8C) and IL-10 (Fig. 8D) were significantly reversed by HMGB1 overexpression. The decreased phosphorylation of NF- $\mathrm{KB}$ 

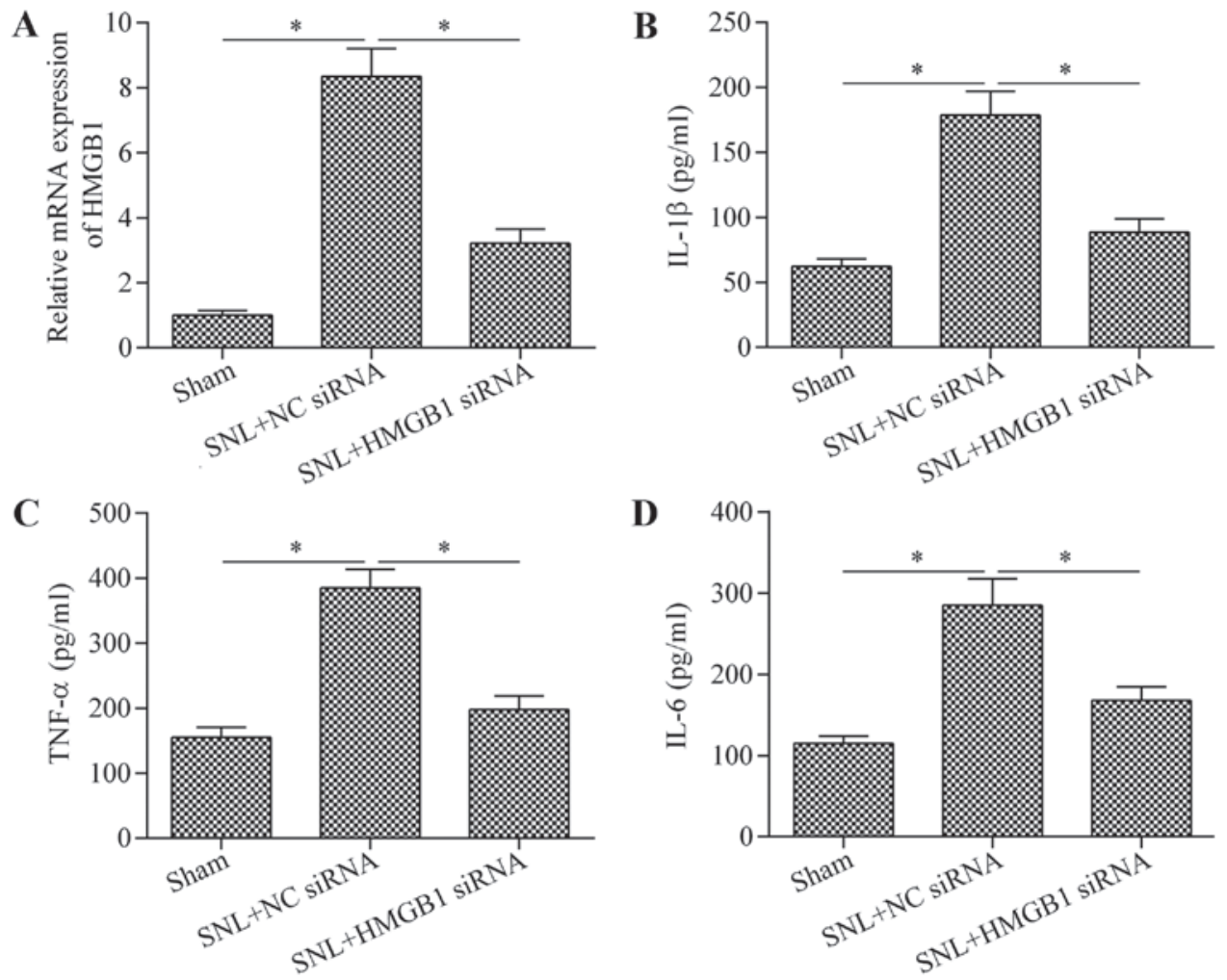

Figure 7. Knockdown of HMGB1 inhibits neuroinflammation in mice with SNL. SNL mice were treated with HMGB1 siRNA and the L3-L5 dorsal root ganglion of mice were harvested on postoperative day 14. NC siRNA served as a control. (A) HMGB1 expression was detected by reverse transcription-quantitative polymerase chain reaction analysis. Protein expression levels of (B) IL-1 $\beta,(C)$ TNF- $\alpha$ and (D) IL-6 were determined by ELISA. $n=3$. "P<0.05 as indicated. HMGB1, high mobility group box 1; SNL, spinal nerve ligation; NC, negative control; siRNA, small interfering RNA; TNF, tumor necrosis factor; IL, interleukin; ELISA, enzyme-linked immunosorbent assay.
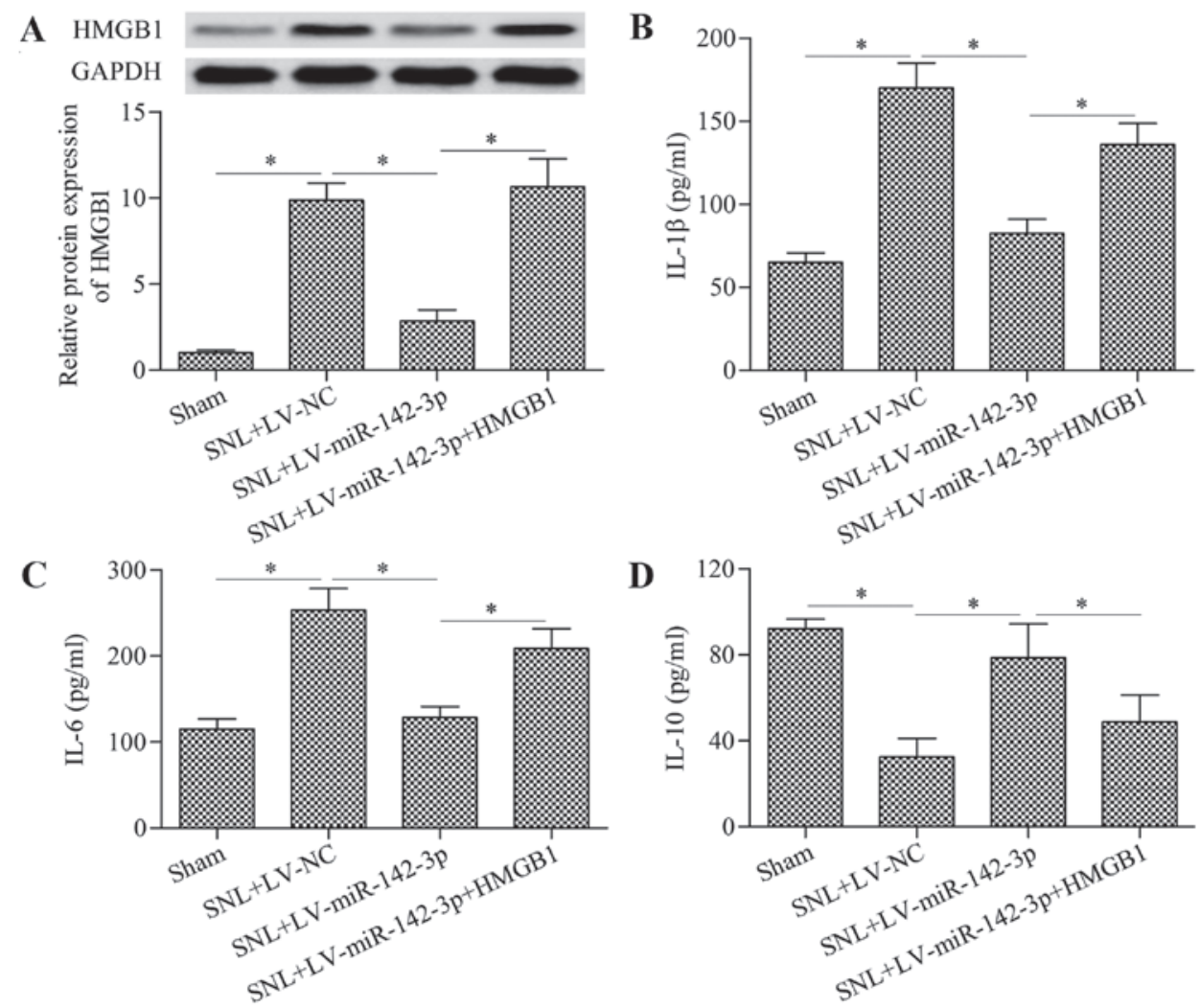

Figure 8. Overexpression of HMGB1 reverses the inhibitory effect of miR-142-3p on neuroinflammation. Mice with SNL were co-infected with LV-miR-142-3p and LV-HMGB1 (without 3'-untranslated region). (A) The protein expression levels of HMGB1 were detected by western blot analysis on postoperative day 14. The levels of (B) IL-1 $\beta$, (C) IL-6 and (D) IL-10 were detected by ELISA. $n=3$. "P<0.05 as indicated. HMGB1, high mobility group box 1; miR, microRNA; SNL, spinal nerve ligation; NC, negative control; LV, lentiviral vector; IL, interleukin; ELISA, enzyme-linked immunosorbent assay. 


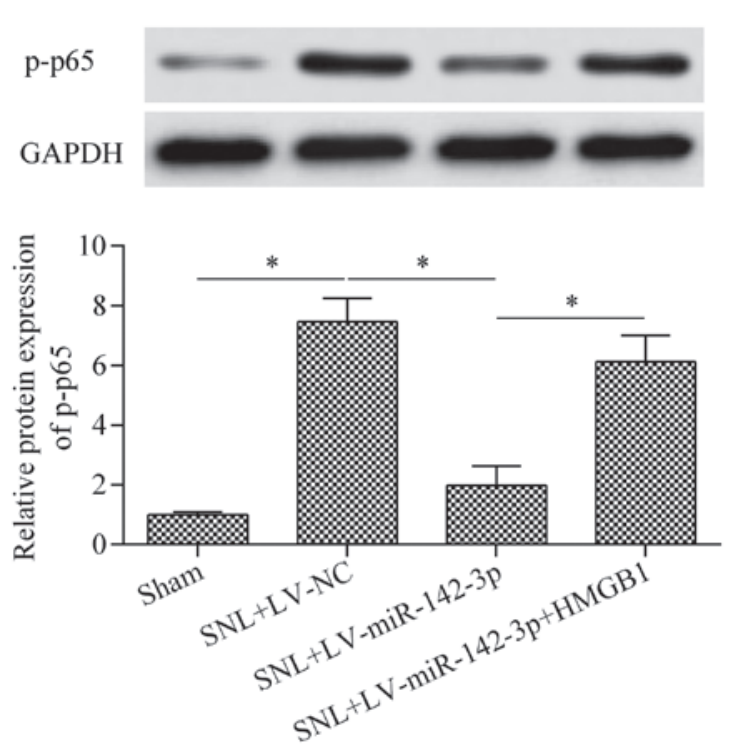

Figure 9. Overexpression of HMGB1 reverses the inhibitory effect of miR-142-3p on NF- $\kappa$ B. Mice with SNL were co-infected with LV-miR-142-3p and LV-HMGB1 (without 3'-untranslated region). The phosphorylation level of p-p65 protein was determined by western blot analysis ( $\mathrm{n}=3$ ). ${ }^{*} \mathrm{P}<0.05$ as indicated. HMGB1, high mobility group box 1 ; miR, microRNA; SNL, spina nerve ligation; NC, negative control; LV, lentiviral vector; $\mathrm{p}$, phosphorylated.

p65 protein in mice with SNL were also significantly restored by HMGB1 overexpression compared with the level in the SNL + LV-miR-142-3p group (Fig. 9). Furthermore, the inhibitory effect of miR-142-3p overexpression on neuropathic pain was also significantly reversed by HMGB1 overexpression at postoperative days 3, 7,14 and 21 in SNL + LV-miR-142-3p + HMGB1 group as compared with the SNL + LV-miR-142-3p group (Fig. 10). Overall, these results suggest that miR-142-3p inhibits neuropathic pain and neuroinflammation by downregulation of HMGB1.

\section{Discussion}

In recent years, miRNA have emerged as critical regulators of persistent neuropathic pain initiation and development (35). There is evidence that miRNA-based therapy may be a promising method for preventing neuropathic pain $(36,37)$. In the present study, the results demonstrated that miR-142-3p functions as a novel miRNA involved in neuropathic pain. The present findings indicated that miR-142-3p negatively regulated neuropathic pain through targeting HMGB1-mediated neuroinflammation. These findings suggest that miR-142-3p may serve as a novel and promising molecular target for the development of anti-neuropathic pain therapies.

There is growing evidence that miRNA serve an important role in the pathogenesis of neuropathic pain progression (6,7,38). A study by Shi et al (39) reported that miR-195 induced by SNL aggravated neuropathic pain by inhibiting Atg14. Suppression of miR-155, miR-19a or miR-221 inhibited neuropathic pain through targeting suppressor of cytokine signaling $1(11,40,41)$. Intrathecal injection of miR-96 and miR-183 alleviated neuropathic pain by downregulation of sodium channel Nav1.3 expression $(42,43)$. Inhibiting sodium channel Nav1.7 by miR-30b inhibited neuropathic pain in nerve injury-induced neuropathic pain in a rat model (44). Reduced
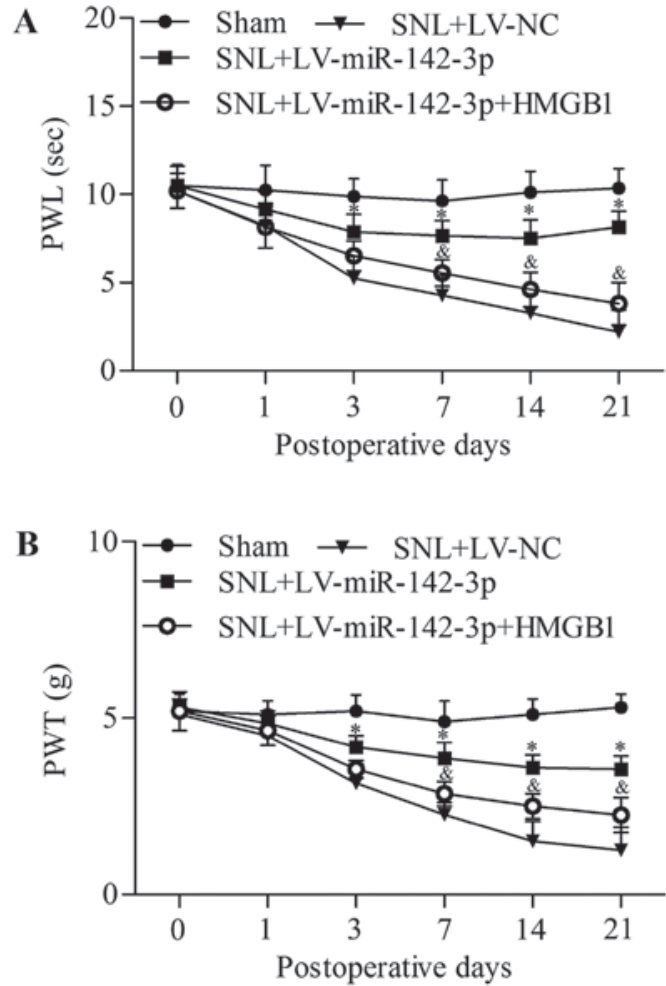

Figure 10. Overexpression of HMGB1 reverses the inhibitory effect of miR-142-3p on neuropathic pain. Mice with SNL were co-infected with LV-miR-142-3p and LV-HMGB1 (without 3'-untranslated region). (A) Thermal hyperalgesia was assessed by measuring the PWL in response to radiant heat stimulation. (B) Mechanical allodynia was assessed by measuring the PWT in response to Von Frey hair stimulation. $n=6$. $^{*} \mathrm{P}<0.05$ vs. SNL + LV-NC; ${ }^{\&} \mathrm{P}<0.05$ vs. SNL + LV-miR-142-3p. HMGB1, high mobility group box 1 ; miR, microRNA; SNL, spinal nerve ligation; NC, negative control; LV, lentiviral vector; PWL, paw withdrawallatency; PWT, paw withdrawal threshold.

miR-203 expression contributed to neuropathic pain through targeting Rap1a (10). Overexpression of miR-146a-5p alleviated neuropathic pain via suppressing TNF receptor-associated factor-6-mediated neuroinflammation (45). Furthermore, recent studies have demonstrated that miR-144, miR-132-3p, miR-124a and miR-15b are also involved in the regulation of neuropathic pain development (46-49). Together, these results suggest that miRNA are critical regulators of neuropathic pain and represent novel and promising targets for the treatment of neuropathic pain. The present study demonstrated that miR-142-3p is a novel miRNA involved in neuropathic pain development.

$\mathrm{NF}-\kappa \mathrm{B}$ is a group of transcription factors that regulate various genes involved in inflammation, apoptosis, angiogenesis and cancer metastasis $(50,51)$. Research has indicated that NF- $\kappa \mathrm{B}$ is involved in the pathogenesis of neuropathic pain through regulating pro-inflammatory cytokine gene expression (52). Inhibition of NF- $\mathrm{kB}$ p65 protein expression significantly attenuates chronic constriction injury-induced mechanical allodynia and thermal hyperalgesia (53). Specific participants in NF- $\kappa B$ signaling transduction have been suggested as molecular targets for treatment of neuropathic pain (54). In the present study, it was demonstrated that miR-142-3p inhibited the activation of NF- $\kappa B$ and expression of pro-inflammatory cytokines, suggesting that miR-142-3p may be a potential modulator of NF- $\kappa \mathrm{B}$ signaling transduction. 
miR-142-3p has been suggested as an inflammation-related miRNA that is involved in various inflammatory diseases, including osteoarthritis (32), ulcerative colitis (55), dermatitis (56), psoriasis (57), keratitis (58) and periodontitis (59). miR-142-3p has been shown to negatively regulate inflammation by suppressing proinflammatory mediators, including NF- $\kappa$ B, TNF- $\alpha$ and IL-6, in macrophages (30). Furthermore, miR-142-3p inhibits the production of inflammatory mediators, including TNF- $\alpha$ and IL-6, in human myeloid inflammatory cells (31), suggesting an anti-inflammatory role of miR-142-3p. The present study demonstrated that miR-142-3p inhibited the production of TNF- $\alpha$, IL- $1 \beta$ and IL- 6 , supporting an anti-inflammatory role for miR-142-3p. Recent studies have reported that miR-142-3p inhibited inflammation by targeting HMGB1 $(32,60)$. A study by Yuan et al $(60)$ reported that peroxisome proliferator-activated receptor $\gamma$ inhibited inflammation associated with the upregulation of miR-142-3p, which targeted and inhibited HMGB1. A study by Wang et al (32) reported that miR-142-3p suppressed NF- $\kappa$ B, TNF- $\alpha$ and IL- 6 in a murine model of osteoarthritis by targeting HMGB1. Additionally, miR-142-3p targets HMGB1 to inhibit hypoxia/reoxygenation-induced cardiomyocyte apoptosis and fibrosis (61). miR-142-3p is also reported to inhibit tumor growth of lung cancer through targeting HMGB1 (62). The present results are consistent with these findings by identifying HMGB1 as a functional target gene of miR-142-3p.

There is increasing evidence that HMGB1 is an important regulator of neuropathic pain $(25,26,63,64)$, and HMGB-1 has been suggested as a promising therapeutic target for the treatment of neuropathic pain $(28,29)$. Inhibition of HMGB1 by antibody neutralization effectively improved pain-related behaviors in animal models of neuropathic pain $(27,65)$. Administration of melatonin, Tanshinone IIA and IL-10 attenuated neuropathic pain development by downregulating HMGB1 (66-69). Furthermore, a recent study indicated that miR-141 inhibits neuropathic pain development by targeting HMGB1 (46), suggesting that targeting HMGB1 using miRNA may serve as a novel therapeutic strategy for the treatment of neuropathic pain. The present study demonstrated that miR-142-3p targeted the 3'-UTR of HMGB1 to regulate HMGB1 expression, providing a novel therapeutic target for the development of miRNA/HMGB1-based therapy for neuropathic pain.

In conclusion, the results of the present study demonstrate that miR-142-3p alleviates neuropathic pain through the downregulation of HMGB1-mediated neuroinflammation. The decreased expression of miR-142-3p leads to the upregulation of HMGB1, which contributes to the development and progression of neuropathic pain. The present study provides novel insight into understanding the molecular pathogenesis of neuropathic pain. Inhibition of HMGB1 by miR-142-3p may serve as a novel therapeutic strategy for preventing neuropathic pain.

\section{References}

1. Baron R: Peripheral neuropathic pain: From mechanisms to symptoms. Clin J Pain 16 (Suppl 2): S12-S20, 2000.

2. Sorge RE, Trang T, Dorfman R, Smith SB, Beggs S, Ritchie J, Austin JS, Zaykin DV, Vander Meulen H, Costigan M, et al: Genetically determined $\mathrm{P} 2 \mathrm{X} 7$ receptor pore formation regulates variability in chronic pain sensitivity. Nat Med 18: 595-599, 2012 .
3. Neville A, Peleg R, Singer Y, Sherf M and Shvartzman P: Chronic pain: A population-based study. Isr Med Assoc J 10: 676-680, 2008

4. O'Connor AB and Dworkin RH: Treatment of neuropathic pain: An overview of recent guidelines. Am J Med 122 (Suppl 10): S22-S32, 2009.

5. Winter J, Jung S, Keller S, Gregory RI and Diederichs S: Many roads to maturity: microRNA biogenesis pathways and their regulation. Nat Cell Biol 11: 228-234, 2009

6. von Schack D, Agostino MJ, Murray BS, Li Y, Reddy PS, Chen J, Choe SE, Strassle BW, Li C, Bates B, et al: Dynamic changes in the microRNA expression profile reveal multiple regulatory mechanisms in the spinal nerve ligation model of neuropathic pain. PLoS One 6: e17670, 2011.

7. Aldrich BT, Frakes EP, Kasuya J, Hammond DL and Kitamoto T: Changes in expression of sensory organ-specific microRNAs in rat dorsal root ganglia in association with mechanical hypersensitivity induced by spinal nerve ligation. Neuroscience 164: 711-723, 2009.

8. Sakai A and Suzuki H: Emerging roles of microRNAs in chronic pain. Neurochem Int 77: 58-67, 2014.

9. Chang HL, Wang HC, Chunag YT, Chou CW, Lin IL, Lai CS, Chang LL and Cheng KI: miRNA expression change in dorsal root ganglia after peripheral nerve injury. J Mol Neurosci 61: 169-177, 2017.

10. Li H, Huang Y, Ma C, Yu X, Zhang Z and Shen L: MiR-203 involves in neuropathic pain development and represses Rapla expression in nerve growth factor differentiated neuronal PC12 cells. Clin J Pain 31: 36-43, 2015.

11. Tan Y, Yang J, Xiang K, Tan Q and Guo Q: Suppression of microRNA-155 attenuates neuropathic pain by regulating SOCS1 signalling pathway. Neurochem Res 40: 550-560, 2015.

12. Moss A, Beggs S, Vega-Avelaira D, Costigan M, Hathway GJ, Salter MW and Fitzgerald M: Spinal microglia and neuropathic pain in young rats. Pain 128: 215-224, 2007.

13. Miljanich G, Rauck R and Saulino M: Spinal mechanisms of pain and analgesia. Pain Pract 13: 114-130, 2013.

14. Scholz J and Woolf CJ: The neuropathic pain triad: Neurons, immune cells and glia. Nat Neurosci 10: 1361-1368, 2007.

15. Moalem G and Tracey DJ: Immune and inflammatory mechanisms in neuropathic pain. Brain Res Brain Res Rev 51: 240-264, 2006

16. Shen W, Hu XM, Liu YN, Han Y, Chen LP, Wang CC and Song C: CXCL12 in astrocytes contributes to bone cancer pain through CXCR4-mediated neuronal sensitization and glial activation in rat spinal cord. J Neuroinflammation 11: 75, 2014.

17. Lu B, Wang C, Wang M, Li W, Chen F, Tracey KJ and Wang H: Molecular mechanism and therapeutic modulation of high mobility group box 1 release and action: An updated review. Expert Rev Clin Immunol 10: 713-727, 2014.

18. Andersson U and Tracey KJ: HMGB1 in sepsis. Scand J Infect Dis 35: 577-584, 2003.

19. Li ZC, Cheng GQ, Hu KZ, Li MQ, Zang WP, Dong YQ, Wang WL and Liu ZD: Correlation of synovial fluid HMGB-1 levels with radiographic severity of knee osteoarthritis. Clin Invest Med 34: E298, 2011.

20. Mantell LL, Parrish WR and Ulloa L: Hmgb-1 as a therapeutic target for infectious and inflammatory disorders. Shock 25: 4-11, 2006.

21. Basta G: Receptor for advanced glycation endproducts and atherosclerosis: From basic mechanisms to clinical implications. Atherosclerosis 196: 9-21, 2008.

22. den Dekker WK, Cheng C, Pasterkamp G and Duckers HJ: Toll-like receptor 4 in atherosclerosis and plaque destabilization. Atherosclerosis 209: 314-320, 2010.

23. Friggeri A, Yang Y, Banerjee S, Park YJ, Liu G and Abraham E: HMGB1 inhibits macrophage activity in efferocytosis through binding to the alphavbeta3-integrin. Am J Physiol Cell Physiol 299: C1267-C1276, 2010.

24. Andersson U, Erlandsson-Harris H, Yang $\mathrm{H}$ and Tracey KJ: HMGB1 as a DNA-binding cytokine. J Leukoc Biol 72: 1084-1091, 2002.

25. Chacur M, Milligan ED, Gazda LS, Armstrong C, Wang H, Tracey KJ, Maier SF and Watkins LR: A new model of sciatic inflammatory neuritis (SIN): Induction of unilateral and bilateral mechanical allodynia following acute unilateral peri-sciatic immune activation in rats. Pain 94: 231-244, 2001.

26. Shibasaki M, Sasaki M, Miura M, Mizukoshi K, Ueno H, Hashimoto S, Tanaka Y and Amaya F: Induction of high mobility group box-1 in dorsal root ganglion contributes to pain hypersensitivity after peripheral nerve injury. Pain 149: 514-521, 2010. 
27. Otoshi K, Kikuchi S, Kato K, Sekiguchi M and Konno S: Anti-HMGB1 neutralization antibody improves pain-related behavior induced by application of autologous nucleus pulposus onto nerve roots in rats. Spine 36: E692-E698, 2011

28. Maeda T, Ozaki M, Kobayashi Y, Kiguchi N and Kishioka S: HMGB1 as a potential therapeutic target for neuropathic pain. J Pharmacol Sci 123: 301-305, 2013.

29. Wan W, Cao L, Khanabdali R, Kalionis B, Tai X and Xia S: The emerging role of HMGB1 in neuropathic pain: A potential therapeutic target for neuroinflammation. J Immunol Res 2016: 6430423,2016

30. Xu G, Zhang Z, Wei J, Zhang Y, Zhang Y, Guo L and Liu X: microR-142-3p down-regulates IRAK-1 in response to Mycobacterium bovis BCG infection in macrophages. Tuberculosis (Edinb) 93: 606-611, 2013

31. Naqvi AR, Fordham JB and Nares S: miR-24, miR-30b, and miR-142-3p regulate phagocytosis in myeloid inflammatory cells. J Immunol 194: 1916-1927, 2015.

32. Wang X, Guo Y, Wang C, Yu H, Yu X and Yu H: MicroRNA-142-3p Inhibits chondrocyte apoptosis and inflammation in osteoarthritis by targeting HMGB1. Inflammation 39: 1718-1728, 2016.

33. Huang ZJ, Li HC, Cowan AA, Liu S, Zhang YK and Song XJ: Chronic compression or acute dissociation of dorsal root ganglion induces cAMP-dependent neuronal hyperexcitability through activation of PAR2. Pain 153: 1426-1437, 2012

34. Livak KJ and Schmittgen TD: Analysis of relative gene expression data using real-time quantitative PCR and the 2(- $\Delta \Delta \mathrm{C}(\mathrm{T}))$ Method. Methods 25: 402-408, 2001.

35. Norcini M, Sideris A, Martin Hernandez LA, Zhang J, Blanck TJ and Recio-Pinto E: An approach to identify microRNAs involved in neuropathic pain following a peripheral nerve injury. Front Neurosci 8: 266, 2014.

36. Jiangpan P, Qingsheng M, Zhiwen Y and Tao Z: Emerging role of microRNA in neuropathic pain. Curr Drug Metab 17: 336-344, 2016.

37. Tan PH, Pao YY, Cheng JK, Hung KC and Liu CC: MicroRNA-based therapy in pain medicine: Current progress and future prospects. Acta Anaesthesiol Taiwan 51: 171-176, 2013.

38. Kusuda R, Cadetti F, Ravanelli MI, Sousa TA, Zanon S, De Lucca FL and Lucas G: Differential expression of microRNAs in mouse pain models. Mol Pain 7: 17, 2011.

39. Shi G, Shi J, Liu K, Liu N, Wang Y, Fu Z, Ding J, Jia L and Yuan W: Increased miR-195 aggravates neuropathic pain by inhibiting autophagy following peripheral nerve injury. Glia 61: 504-512, 2013.

40. Wang C, Jiang Q, Wang M and Li D: MiR-19a targets suppressor of cytokine signaling 1 to modulate the progression of neuropathic pain. Int J Clin Exp Pathol 8: 10901-10907, 2015.

41. Xia L, Zhang Y and Dong T: Inhibition of microRNA-221 alleviates neuropathic pain through targeting suppressor of cytokine signaling 1. J Mol Neurosci 59: 411-420, 2016.

42. Chen HP, Zhou W, Kang LM, Yan H, Zhang L, Xu BH and Cai WH: Intrathecal miR-96 inhibits Nav1.3 expression and alleviates neuropathic pain in rat following chronic construction injury. Neurochem Res 39: 76-83, 2014

43. Lin CR, Chen KH, Yang CH, Huang HW and Sheen-Chen SM Intrathecal miR-183 delivery suppresses mechanical allodynia in mononeuropathic rats. Eur J Neurosci 39: 1682-1689, 2014.

44. Shao J, Cao J, Wang J, Ren X, Su S, Li M, Li Z, Zhao Q and Zang W: MicroRNA-30b regulates expression of the sodium channel Nav1.7 in nerve injury-induced neuropathic pain in the rat. Mol Pain: Oct 19,2016 (Epub ahead of print). doi: 10.1177/1744806916671523.

45. Lu Y, Cao DL, Jiang BC, Yang T and Gao YJ: MicroRNA-146a-5p attenuates neuropathic pain via suppressing TRAF6 signaling in the spinal cord. Brain Behav Immun 49: 119-129, 2015.

46. Zhang J, Zhang H and Zi T: Overexpression of microRNA-141 relieves chronic constriction injury-induced neuropathic pain via targeting high-mobility group box 1 . Int J Mol Med 36: $1433-1439,2015$

47. Leinders M, Üceyler N, Pritchard RA, Sommer C and Sorkin LS: Increased miR-132-3p expression is associated with chronic neuropathic pain. Exp Neurol 283: 276-286, 2016.

48. Heyn J, Luchting B, Hinske LC, Hübner M, Azad SC and Kreth S miR-124a and miR-155 enhance differentiation of regulatory $\mathrm{T}$ cells in patients with neuropathic pain. J Neuroinflammation 13 : 248, 2016.

49. Ito N, Sakai A, Miyake N, Maruyama M, Iwasaki H, Miyake K, Okada T, Sakamoto A and Suzuki H: miR-15b mediates oxaliplatin-induced chronic neuropathic pain through BACE1 down-regulation. Br J Pharmacol 174: 386-395, 2017.
50. Neumann $\mathbf{M}$ and Naumann M: Beyond IkappaBs: Alternative regulation of NF-kappaB activity. FASEB J 21: 2642-2654, 2007.

51. Maeda S and Omata M: Inflammation and cancer: Role of nuclear factor-kappaB activation. Cancer Sci 99: 836-842, 2008.

52. Sakaue G, Shimaoka M,Fukuoka T, Hiroi T, Inoue T, Hashimoto N, Sakaguchi T, Sawa Y, Morishita R, Kiyono H, et al: NF-kappa B decoy suppresses cytokine expression and thermal hyperalgesia in a rat neuropathic pain model. Neuroreport 12: 2079-2084, 2001.

53. Sun T, Song WG, Fu ZJ, Liu ZH, Liu YM and Yao SL: Alleviation of neuropathic pain by intrathecal injection of antisense oligonucleotides to p65 subunit of NF-kappaB. Br J Anaesth 97: 553-558, 2006

54. Niederberger $E$ and Geisslinger G: The IKK-NF-kappaB pathway: A source for novel molecular drug targets in pain therapy? FASEB J 22: 3432-3442, 2008

55. Schaefer JS, Attumi T, Opekun AR, Abraham B, Hou J, Shelby H, Graham DY, Streckfus C and Klein JR: MicroRNA signatures differentiate Crohn's disease from ulcerative colitis. BMC Immunol 16: 5, 2015.

56. Ralfkiaer U, Lindahl LM, Litman T, Gjerdrum LM, Ahler CB, Gniadecki R, Marstrand T, Fredholm S, Iversen L, Wasik MA, et al: MicroRNA expression in early mycosis fungoides is distinctly different from atopic dermatitis and advanced cutaneous T-cell lymphoma. Anticancer Res 34: 7207-7217, 2014

57. Pivarcsi A, Meisgen F, Xu N, Ståhle M and Sonkoly E: Changes in the level of serum microRNAs in patients with psoriasis after antitumour necrosis factor- $\alpha$ therapy. Br J Dermatol 169: 563-570, 2013.

58. Boomiraj H, Mohankumar V, Lalitha P and Devarajan B: Human corneal microRNA expression profile in fungal keratitis. Invest Ophthalmol Vis Sci 56: 7939-7946, 2015.

59. Perri R, Nares S, Zhang S, Barros SP and Offenbacher S MicroRNA modulation in obesity and periodontitis. J Dent Res 91: 33-38, 2012

60. Yuan Z, Luo G, Li X, Chen J, Wu J and Peng Y: PPAR $\gamma$ inhibits HMGB1 expression through upregulation of miR-142-3p in vitro and in vivo. Cell Signal 28: 158-164, 2016.

61. Wang Y, Ouyang M, Wang Q and Jian Z: MicroRNA-142-3p inhibits hypoxia/reoxygenation induced apoptosis and fibrosis of cardiomyocytes by targeting high mobility group box 1 . Int J Mol Med 38: 1377-1386, 2016.

62. Xiao P and Liu WL: MiR-142-3p functions as a potential tumor suppressor directly targeting HMGB1 in non-small-cell lung carcinoma. Int J Clin Exp Pathol 8: 10800-10807, 2015.

63. Feldman P, Due MR, Ripsch MS, Khanna R and White FA: The persistent release of HMGB1 contributes to tactile hyperalgesia in a rodent model of neuropathic pain. J Neuroinflammation 9: 180,2012

64. Zhang FF, Morioka N,Harano S, Nakamura Y,Liu K, Nishibori M, Hisaoka-Nakashima K and Nakata Y: Perineural expression of high-mobility group box-1 contributes to long-lasting mechanical hypersensitivity via matrix metalloproteinase-9 upregulation in mice with painful peripheral neuropathy. J Neurochem: Nov 18 , 2015 (Epub ahead of print). doi: 10.1111/jnc.13434.

65. Nakamura Y,MoriokaN,AbeH,Zhang FF,Hisaoka-NakashimaK, Liu K, Nishibori M and Nakata Y: Neuropathic pain in rats with a partial sciatic nerve ligation is alleviated by intravenous injection of monoclonal antibody to high mobility group box-1. PLoS One 8: e73640, 2013

66. Lin TB, Hsieh MC, Lai CY, Cheng JK, Wang HH, Chau YP, Chen GD and Peng HY: Melatonin relieves neuropathic allodynia through spinal MT2-enhanced PP2Ac and downstream HDAC4 shuttling-dependent epigenetic modification of hmgb1 transcription. J Pineal Res 60: 263-276, 2016.

67. Wang YS, Li YY, Wang LH, Kang Y, Zhang J, Liu ZQ, Wang K, Kaye AD and Chen L: Tanshinone IIA attenuates chronic pancreatitis-induced pain in rats via downregulation of HMGB1 and TRL4 expression in the spinal cord. Pain Physician 18: E615-E628, 2015.

68. Ma YQ, Chen YR, Leng YF and Wu ZW: Tanshinone IIA downregulates HMGB1 and TLR4 expression in a spinal nerve ligation model of neuropathic pain. Evid Based Complement Alternat Med 2014: 639563, 2014.

69. He Z, Guo Q, Xiao M, He C and Zou W: Intrathecal lentivirusmediated transfer of interleukin-10 attenuates chronic constriction injury-induced neuropathic pain through modulation of spinal highmobility group box 1 in rats. Pain Physician 16: E615-E625, 2013. 